\title{
Vulvar Cancer in China: Epidemiological Features and Risk Analysis
}

\author{
Xue Xiao 1, 5, 7,8*, Yi-Bo Meng1,9*, Peng Bai ${ }^{3 *}$, Juan Zou4, Ya Zhang7, Tri M. Bui Nguyen², Jian-Guo Xiao5, \\ Xue-Mei Gao ${ }^{1,5}, 6 \# \bowtie$, Bang-Fen Wen ${ }^{7 \# \bowtie}$ \\ 1. Department of Gynecology and Obstetrics, West China Second University Hospital, Sichuan University, Chengdu 610041, P.R.China; \\ 2. The Oak Ridge Institute for Science and Education, USA; \\ 3. West China School of Preclinical and Forensic Medicine, Sichuan University, Chengdu 610041, P.R. China; \\ 4. Department of Pathology, West China Second University Hospital, Sichuan University, Chengdu 610041, P.R.China; \\ 5. Department of Pathology, University of Texas M. D. Anderson Cancer Center, Houston, Texas; \\ 6. Laboratory of Genetics, West China Institute of Women and Children's Health, West China Second Hospital, Sichuan University, Chengdu 610041, \\ P.R.China; \\ 7. Department of Gynaecology and Obstetrics, ZiYang maternal and child health care hospital, West China Second University Union-hospital, Ziyang, \\ P.R.China; \\ 8. Key Laboratory of Obstetric \& Gynecologic and Pediatric Diseases and Birth Defects of Ministry of Education, Chengdu 610041, P.R.China; \\ 9. Department of Gynaecology and Obstetritics, MeiShan City People's Hospital, Meishan 620010, P.R. China. \\ * Xue Xiao, Yibo Meng and Peng Bai contributed equally to this paper. \\ \# Bangfen Wen and Xuemei Gao contributed equally to this paper.
}

$\square$ Corresponding author: Bang-Fen Wen, Department of Gynaecology and Obstetrics, ZiYang maternal and child health care hospital, West China Second University Union-hospital, No. 82, Heping Road Ziyang, Sichuan, 641300, People's Republic of China. Phone: 86-28-18982989268; Fax: 86-28-26222079. E-mail: baipeng@scu.edu.cn; Xue-Mei Gao, Department of Obstetrics and Gynecology, West China Second University Hospital, Sichuan University, No. 20, Section 3, Renmin Nan Lu Chengdu, Sichuan, 610041, People's Republic of China. Phone: 86-28-15908149408; Fax: 86-28-85559065. E-mail: forensicbp@gmail.com

(c) Ivyspring International Publisher. This is an open access article distributed under the terms of the Creative Commons Attribution (CC BY-NC) license (https://creativecommons.org/licenses/by-nc/4.0/). See http://ivyspring.com/terms for full terms and conditions.

Received: 2017.04.10; Accepted: 2017.05.18; Published: 2017.08.25

\begin{abstract}
Objective: Describe for the first time the clinical, epidemiological features of vulvar cancer in southwest China. Identify risk factors and provide reference for the prevention of vulvar cancer. Method: We retrospectively analyzed 885 patients admitted to the West China Second University Hospital for vulvar diseases between 2006 and 2016. Vulvar cancer patients with previously diagnosed vulvar nonneoplastic epithelial disorders $(n=132)$ were analyzed and compared to those without prior history of vulvar nonneoplastic epithelial disorders $(n=219)$. Comparisons were also made among cancer patients and non-cancer patients with vulvar nonneoplastic epithelial disorders $(n=288)$ and vulvar squamous intraepithelial lesions $(n=246)$. The risk factors leading to vulvar cancer for the patients with vulvar nonneoplastic epithelial disorder were analyzed by univariate analysis. Furthermore, differences of the epidemiological features of vulvar nonneoplastic epithelial disorders, vulvar squamous intraepithelial lesion and vulvar cancer were identified. Results: According to the univariate analysis, age, first coital age, educational level, smoking, history of vaginal atrophy, HPV infection, lesion sites of the upper vulva and histo-pathological changes are strongly positively correlated with vulvar cancer. By comparing the features of vulvar cancer with those of the vulvar nonneoplastic epithelial disorder and vulvar squamous intraepithelial lesion, we found that on average patients with vulvar cancer had the highest age (ranged from 50 to 59), the lowest first coital age and the highest number of pregnancies and births. The incidences of vulvar nonneoplastic epithelial disorder and vulvar cancer were $1 / 1000$ and 2.5/100,000 respectively with an increasing trend during last 10 years. Conclusion: Age, first coital age, educational level, smoking, atrophic vagina history, HPV infection, lesion sites of the upper vulva and histo-pathological changes are the risk factors that lead to vulvar cancer. Vulvar nonneoplastic epithelial disorder, vulvar squamous intraepithelial lesion and vulvar cancer each has distinct epidemiological features. Prompt surgical intervention and subsequent treatments are the key to a better outcome of vulvar cancer.
\end{abstract}

Key words: Vulvar nonneoplastic epithelial disorder, vulvar squamous intraepithelial neoplasia, precancerous lesion, vulvar cancer, hazardous factors, non-conditional logistic regression. 


\section{Introduction}

With pruritus vulvae and hypomelanosis as the main clinical features, vulvar nonneoplastic epithelial disorders refer to a set of chronic degenerative diseases of the vulvar skin mucosa tissues and pigmentogenesis. The types of pathology mainly include lichen simplex chronicus (previously called squamous cell hyperplasia), lichen scleroses and other vulvar skin disease. Vulvar nonneoplastic epithelial disorder has once been considered as the precancerous lesion of vulvar cancer for its unknown pathogeny, long course of disease and high rate of recurrence [1]. Recently it has been reported that vulvar nonneoplastic epithelial disorder has a malignancy rate of $5 \%$ and that the precancerous lesion rate becomes higher if the lichen scleroses evolve into hyperplasia [2].The relationship between vulvar cancer and nonneoplastic epithelial disorder and vulvar squamous intraepithelial lesion has again become the focus of studies since Mitchell MF reported that vulvar infiltrating tumor and vulvar nonneoplastic epithelial disorder had different epidemiological features [3-6].

Unlike cervical cancer which has a well-established correlation with human papillomavirus (HPV) infection, reports on HPV infection rate in vulvar cancer vary with a broad range from $0 \%$ to $85 \%$. Most of those HPV-vulvar cancer studies are of limited value due to small number of cases, variability among study populations, and different methods of detection. It has been suggested that vulvar cancer comprises two completely separate diseases. The first type occurs with high prevalence among older women and develops from nonneoplastic epithelial disorders such as lichen sclerosus. The second type affects predominantly young women and often develops from vulvar intraepithelial neoplasia caused by HPV infection [7, 8].

In this study, 885 patients treated in our hospital with vulvar disease between 2006 and 2016 were retrospectively analyzed. We analyzed the clinical and pathological features and various lab indexes of those patients. The epidemiological features of vulvar nonneoplastic epithelial disorders, vulvar squamous intraepithelial lesion and vulvar cancer were described [9]. Risk factors underlying of vulvar cancer were identified. To confirm HPV infection, a large number of samples were analyzed using a sensitive PCR-based, reverse blot hybridization detection method. This study suggests the importance of taking precautions and interventions at precancerous stage in the prevention of vulvar cancer.

\section{Materials and Methods}

\section{Study Methods and Subjects}

The study subjects were 885 patients with vulvar disease in West China Second University Hospital from 2006 to 2016. 132 cases of patients with vulvar cancer resulted from vulvar nonneoplastic epithelial disorder, 288 cases of patients with vulvar nonneoplastic epithelial disorder, 246 cases of patients with vulvar squamous intraepithelial lesion and 219 cases of patients with vulvar cancer (without vulvar noneoplastic epithelial disorder history) were analyzed. All the cases meet the diagnosis criteria (ISSVD, 2011, Vulvar skin disease clinical classification; ISSVD, 2006, Vulvar skin disease pathological classification) and their vulvar cancers are classified according to the standards made by FIGO 2009. In the classification of female reproductive organs (WHO, 2014) vulvar squamous intraepithelial lesions are classified into low-grade squamous intraepithelial lesion, high-grade squamous intraepithelial lesion and differentiated-type vulvar intraepithelial neoplasia. All the patients had finished pathological examination in the hospital and were screened for HPV infection. The study received local Institutional Review Board approval.

The clinical examinations included routine gynecological examination, cervical and vaginal smear, cytology examination, acetowhitening test, biopsy of the lesion sites and pathological examination. Only cases with both appropriate pathology materials and surgical pathology reports were included in our studies. Paraffin sections of tissue were treated with $0.3 \%$ hydrogen peroxide at room temperature to block endogenous peroxidase activities ensued by a $5 \%$ bovine serum albumin incubation. Treated sections were exposed to antibodies. Images were taken using a Leica DM 4000B photo microscope (Solms; Magnification, $\times 200$ and $\times 400)$. Hematoxylin and eosin $(H \& E)$ were carried out using standard techniques. All cases were reviewed by two surgical pathologists to confirm the diagnosis. Particularly, any vulvar squamous intraepithelial lesion which did not meet the morphologic criteria of Basaloid and Warty carcinoma was designated squamous cell carcinoma, unless otherwise specified.

\section{Diagnosis Standard and Examination}

The diagnoses of different diseases were made according to the examination of the pathological 
tissue after surgery. Cases included in the analyses were carefully differentiated from papilloma, vulvar tuberculosis, vulvar vitiligo and basal cell carcinoma of the skin. Cases of pigmentogenesis resulted from other reasons were excluded from the study after and cross-examinations on materials obtained from different sources. The standard for diagnosis was in accordance with the latest international guidelines.

\section{Study Content}

We investigated 11 potential risk factors based on prior epidemiological studies in other country and other regions in China. Those 11 factors were histo-pathological types of vulvar nonneoplastic epithelial disorders, early first coital age, multiple gestations, educational level, location of the tumor, disease of the uterus, atrophic vagina history, underlying diseases, smoking, age and HPV infection. It has been pointed out that vulvar infiltrating cancers and vulvar nonneoplastic epithelial disorders have different epidemiological features; and that HPV infection has become an important index for above argumentation. Therefore, HPV infection was investigated in particular using PCR in order to depict its relationship with vulvar cancer resulting from vulvar nonneoplastic epithelial disorders. Histo-pathological types of vulvar nonneoplastic epithelial disorders and lesion sites were also analyzed for their association with vulvar cancer [10, $11]$.

\section{HPV Genotyping}

Dissected tissue sections were deparaffinized with xylene, and then rinsed with absolute ethanol, and finally digested with proteinase $\mathrm{K}$ in ATL tissue lysis buffer (DNeasy Tissue Kit; Qiagen, Valencia, CA, USA). Digested tissues were subsequently processed for DNA extraction using the DNeasy Tissue Kit according to the instructions previously. Isolated DNA was subjected to PCR and HPV detection by the Roche LINEAR ARRAY HPV Genotyping Test (Roche Diagnostics, Branchburg, NJ, USA).

\section{Study Subjects}

The information of the study subjects were collected from the patients' files in the hospital. Information was collected using a standardized questionnaire. Classification and proportion of cases with vulvar diseases are shown in Table 2.

\section{Statistical Analyses}

Foxbase system was employed to set up the database. Patient information was logged in after careful examination. Categorical variables such as tumor histology and tumor site were summarized by frequencies and percentages. Continuous variables such as patient age was analyzed by mean, range, and standard deviation (s.d.); $X^{2}$ tests and odds ratios with exact confidence intervals were used to compare categorical variables among valvar cancers with or without HPV infection, presence or absence of smoking and types of tissue histology. Independent two-tailed Student's t-tests were used for comparisons of age. Statistical analyses were carried out using SPSS13.0 software.

\section{Results}

\section{Analyzing the risk factors of vulvar cancer}

Among the 351 vulvar cancer cases treated from 2006 to 2016, 132 patients had a history of vulvar nonneoplastic epithelial disorder, which accounted for $37.61 \%$ of the total vulvar cancer patients. In vulvar carcinoma cases with vulvar nonneoplastic epithelial disorders, $63.64 \%$ of the patients were over 50 years old, $36.36 \%$ had over 3 times of pregnancy, $61.36 \%$ had nonpalpable lymphadenectasis of ilioinguinal lymph, $95.45 \%$ had long-term pruritus vulvae, $59.09 \%$ had pathological changes in clitoris or upper labium, $84.09 \%$ had over $2 \mathrm{~cm}$ lesions, $52 \%$ had vulvar mass with ulcer, and $65.91 \%$ had pathological changes in labium majora, fewer in labia minora and urethra, and the least in bartholin gland and perineum. We analyzed risk factors of vulvar cancer using non-conditional logistic regression model. Factors such as age, HPV infection, sexual intercourse, multiple births, underlying diseases, history of vaginal atrophy, educational level, histo-pathological types and sites of tumor were all taken into consideration. When comparing 288 cases of patients with only vulvar nonneoplastic epithelial disorders to 132 vulvar cancer patients resulted from vulvar nonneoplastic epithelial disorders, the univariate analysis indicated that age, HPV infection, first coital age, multiple gestations $(\geq 3)$, smoking, atrophic vagina history, educational level, upper vulvae, histo-pathological tissue changes were statistical significance $(P<0.05)$ (Table 1$)$.

\section{Differences in epidemiological features among vulvar cancer, vulvar nonneoplastic epithelial disorder and vulvar squamous intraepithelial lesion}

Totally 885 cases with vulvar diseases were included in our study (Table 2). We found that the epidemiological features of vulvar nonneoplastic epithelial disorders, vulvar squamous intraepithelial neoplasia and vulvar cancer are partially different. Differences also existed between vulvar squamous intraepithelial cell lesion and vulva nonneoplasia epithelial disorder. The results are presented in the 
following three sections: 1) Age distribution of different vulvar diseases, 2) Epidemiological features of vulvar nonneoplastic epithelial disorders, vulvar squamous intraepithelial lesion and vulvar cancer, and 3) HPV infection and genotypes in vulvar nonneoplastic epithelial disorders, vulvar squamous intraepithelial neoplasia and vulvar cancer (Tables 3-4).

Table 1. Analyses of hazardous factors for vulvar cancer

\begin{tabular}{|c|c|c|c|c|c|}
\hline $\begin{array}{l}\text { Hazardous } \\
\text { factors }\end{array}$ & $\begin{array}{l}\text { Clinical } \\
\text { information }\end{array}$ & $\begin{array}{l}\text { VC from } \\
\text { NED } \\
(n=132)\end{array}$ & $\begin{array}{l}\text { NED } \\
(n=288)\end{array}$ & $\begin{array}{l}\text { Chi-square } \\
\text { test }\end{array}$ & $P$ value \\
\hline \multirow[t]{3}{*}{ Age } & & & & 33.939 & $<0.001$ \\
\hline & $>50$ & 84 & 96 & & \\
\hline & $<50$ & 48 & 192 & & \\
\hline \multirow{3}{*}{$\begin{array}{l}\text { HPV } \\
\text { infection }\end{array}$} & & & & 26.606 & $<0.001$ \\
\hline & YES & 0 & 51 & & \\
\hline & $\mathrm{NO}$ & 132 & 237 & & \\
\hline \multirow{3}{*}{$\begin{array}{l}\text { Early first } \\
\text { coital age }\end{array}$} & & & & 61.770 & $<0.001$ \\
\hline & YES & 42 & 12 & & \\
\hline & NO & 90 & 276 & & \\
\hline \multirow{3}{*}{$\begin{array}{l}\text { Multiple } \\
\text { gestations } \\
(\geq 3)\end{array}$} & & & & 9.772 & 0.002 \\
\hline & YES & 48 & 63 & & \\
\hline & NO & 84 & 225 & & \\
\hline \multirow[t]{3}{*}{ Smoking } & & & & 20.193 & $<0.001$ \\
\hline & YES & 36 & 42 & & \\
\hline & NO & 96 & 246 & & \\
\hline \multirow{3}{*}{$\begin{array}{l}\text { Underlying } \\
\text { diseases }\end{array}$} & & & & 0.000 & 1.000 \\
\hline & hypertension & 9 & 21 & & \\
\hline & diabetes & 3 & 6 & & \\
\hline \multirow{3}{*}{$\begin{array}{l}\text { Atrophic } \\
\text { vagina }\end{array}$} & & & & 14.073 & $<0.001$ \\
\hline & YES & 81 & 120 & & \\
\hline & NO & 51 & 168 & & \\
\hline \multirow{4}{*}{$\begin{array}{l}\text { Disease of } \\
\text { the uterus }\end{array}$} & & & & 8.250 & 0.016 \\
\hline & uterine leiomyoma & 6 & 21 & & \\
\hline & cervical carcinoma & 3 & 0 & & \\
\hline & CIN & 3 & 3 & & \\
\hline \multirow{3}{*}{$\begin{array}{l}\text { Education } \\
\text { level (grade) }\end{array}$} & & & & 50.293 & $<0.001$ \\
\hline & $>12$ & 0 & 87 & & \\
\hline & $<12$ & 132 & 201 & & \\
\hline \multirow{4}{*}{$\begin{array}{l}\text { Pathological } \\
\text { type }\end{array}$} & & & & 25.313 & $<0.001$ \\
\hline & $\begin{array}{l}\text { lichen simplex } \\
\text { chronicus }\end{array}$ & 102 & 150 & & \\
\hline & lichen sclerosus & 18 & 63 & & \\
\hline & other & 12 & 75 & & \\
\hline \multirow{4}{*}{$\begin{array}{l}\text { Location of } \\
\text { the mixed } \\
\text { tumor }\end{array}$} & & & & 35.383 & $<0.001$ \\
\hline & upper & 78 & 84 & & \\
\hline & middle & 30 & 132 & & \\
\hline & lower & 24 & 72 & & \\
\hline
\end{tabular}

VC from NED: Vulvar cancer resulted from nonneoplastic epithelial disorder; NED: Vulvar nonneoplastic epithelial disorder.
Table 2. Classification and proportion of 885 cases of patients with vulvar disease

\begin{tabular}{lll}
\hline Item & $\begin{array}{l}\text { Number of Cases } \\
\text { (Total 885) }\end{array}$ & \\
\hline $\begin{array}{l}\text { Vulvar nonneoplastic epithelial disorder } \\
\text { Lichen simplex chronicus (called squamous }\end{array}$ & 288 & $32.54 \%$ \\
cell hyperplasia previously) & 63 & $7.12 \%$ \\
$\begin{array}{l}\text { lichen sclerosus } \\
\text { other }\end{array}$ & 150 & $16.95 \%$ \\
vulvar squamous intraepithelial neoplasia & 75 & $8.47 \%$ \\
Low-grade squamous intraepithelial lesion, & 36 & $27.80 \%$ \\
LSIL & & $4.07 \%$ \\
High-grade squamous intraepithelial lesion, & 102 & $11.53 \%$ \\
HSIL & & \\
$\begin{array}{l}\text { Differentiated-type vulvar intraepithelial } \\
\text { neoplasia }\end{array}$ & 108 & $12.20 \%$ \\
Vulvar cancer (Vulvar squamous cell & 351 & $39.66 \%$ \\
carcinoma) & & \\
I & 195 & $22.03 \%$ \\
II & 83 & $9.38 \%$ \\
III & 42 & $4.75 \%$ \\
IV & 31 & $3.50 \%$ \\
Type of pathological tissue(nonneoplastic & 132 & $14.92 \%$ \\
pathology before vulvar cancer) & & \\
lichen simplex chronicus & 102 & $11.53 \%$ \\
lichen sclerosus & 18 & $2.03 \%$ \\
other & 12 & $1.36 \%$ \\
Vulvar cancer without nonneoplastic & 219 & $24.75 \%$ \\
disorder history & & \\
\hline
\end{tabular}

\section{Age distribution of different vulvar diseases.}

There was a distinct difference among the proportion of the patients over 50 years old in patients with vulvar cancer, vulvar nonneoplastic epithelial disorders and vulvar squamous intraepithelial lesion. Our study showed a vulvar cancer peaked among the patients whose ages ranged from 50 to 59 years of age while the vulvar nonneoplastic epithelial disorder peak appeared among the patients from 40 to 59 years of age. The average age of the patients in the vulvar cancer with nonneoplastic epithelial disorders was 51.9 years old while in the vulvar nonneoplastic epithelial disorders group was 45.9 years old. With 60 years old as a boundary for the risk factors, foreign studies reported that the incidence of vulvar cancer was in direct proportion with age among women over 60 years old. It was also reported that the number of patients with vulvar cancer was the largest among the women aged from 70 to 79 years old in Greece [12]. However, our analysis showed that the peak period of vulvar cancer was younger than 60 years old in China. Besides that, our study shows that the average age of the patients with vulvar cancer was the highest and the age of the patients with intraepithelial neoplasia was the lowest. The incidence of HSIL decreased with age. The difference in age may be associated with the fact that young women are more susceptible to HPV infection. 
Table 3. The epidemiological features of vulvar nonneoplastic epithelial disorder, vulvar squamous intraepithelial lesion and vulvar cancer

\begin{tabular}{|c|c|c|c|c|c|c|}
\hline \multirow[t]{2}{*}{ Variables } & \multicolumn{2}{|l|}{ Vulvar Cancer } & \multirow[t]{2}{*}{ VSIL } & \multirow[t]{2}{*}{ NED } & \multirow[t]{2}{*}{$x^{2}$} & \multirow[t]{2}{*}{$P$ value } \\
\hline & VC from NED & VC from HSIL & & & & \\
\hline Number & 132 & 219 & 246 & 288 & & \\
\hline Age & $51.9 \pm 16$ & $50.20 \pm 15$ & $42.71 \pm 15$ & $45.90 \pm 12$ & & \\
\hline Early first coital age & $20 \pm 3$ & $20 \pm 4$ & $23 \pm 2$ & $22 \pm 3$ & & \\
\hline Pregnant times & $4.27 \pm 2.25$ & $4.02 \pm 3.22$ & $2.59 \pm 1.23$ & $2.87 \pm 2.01$ & & \\
\hline Gestation times & $3.25 \pm 2.07$ & $3.09 \pm 3.01$ & $1.66 \pm 1.34$ & $1.61 \pm 2.21$ & & \\
\hline \multicolumn{7}{|c|}{ Location of the mixed tumor } \\
\hline Upper & $53.79 \%(71 / 132)$ & $16.89 \%(37 / 219)$ & $13.00 \%(32 / 246)$ & $11.81 \%(34 / 288)$ & 117.665 & $<0.001$ \\
\hline Middle & $18.94 \%(25 / 132)$ & $33.79 \%(74 / 219)$ & $34.15 \%(84 / 246)$ & $31.94 \%(92 / 288)$ & & \\
\hline Lower & $27.27 \%(36 / 132)$ & $49.32 \%(108 / 219)$ & $50.78 \%(130 / 246)$ & $56.25 \%(162 / 288)$ & & \\
\hline Uterine leiomyoma & $4.55 \%(6 / 132)$ & $6.85(15 / 219)$ & $7.32(18 / 246)$ & $7.29 \%(21 / 288)$ & 1.276 & 0.735 \\
\hline Atrophic vagina & $61.36 \%(81 / 132)$ & $57.08(125 / 219)$ & $44.72(110 / 246)$ & $41.67 \%(120 / 288)$ & 21.768 & $<0.001$ \\
\hline HPV Positive & $0(0 / 132)$ & $70.78(155 / 219)$ & $83.74(206 / 246)$ & $17.71 \%(51 / 288)$ & 399.648 & $<0.001$ \\
\hline $\begin{array}{l}\text { Operation history of } \\
\text { vulva }\end{array}$ & $26.52(35 / 132)$ & $23.74 \%(52 / 219)$ & $4.47 \%(11 / 246)$ & $2.78 \%(8 / 288)$ & 91.488 & $<0.001$ \\
\hline Malignant history & $2.27 \%(3 / 132)$ & $2.74 \%(6 / 219)$ & $4.47 \%(11 / 246)$ & $1.04 \%(3 / 288)$ & 6.240 & 0.101 \\
\hline Hypertention & $12.12 \%(16 / 132)$ & $12.79 \%(28 / 219)$ & $8.94 \%((22 / 246)$ & $2.08 \%(6 / 288)$ & 23.471 & $<0.001$ \\
\hline $\mathrm{DM}$ & $15.15 \%(20 / 132)$ & $15.98 \%(35 / 219)$ & $4.47 \%(11 / 246)$ & $3.82 \%(11 / 288)$ & 35.708 & $<0.001$ \\
\hline Obsity & $38.64 \%(51 / 132)$ & $36.99 \%(81 / 219)$ & $36.59 \%(90 / 246)$ & $23.96 \%(69 / 288)$ & 15.573 & $<0.001$ \\
\hline
\end{tabular}

VC from NED: Vulvar cancer resulted from nonneoplastic epithelial disorder; VC from HSIL: Vulvar cancer resulted from High-grade squamous intraepithelial lesion; VSIL: vulva squamous intraepithelial lesion; NED: Vulvar nonneoplastic epithelial disorder.

Table 4. The epidemiological features of vulvar cancer

\begin{tabular}{|c|c|c|c|c|}
\hline \multirow[t]{2}{*}{ Variables } & \multicolumn{2}{|l|}{ Vulvar Cancer } & \multirow[t]{2}{*}{$x^{2}$} & \multirow[t]{2}{*}{$P$ value } \\
\hline & $\begin{array}{l}\text { VC from NED } \\
(132)\end{array}$ & $\begin{array}{l}\text { VC from HSIL } \\
(219)\end{array}$ & & \\
\hline Age & $51.9 \pm 16$ & $50.20 \pm 15$ & & \\
\hline $\begin{array}{l}\text { Early first coital } \\
\text { age }\end{array}$ & $20 \pm 3$ & $20 \pm 4$ & & \\
\hline Pregnant times & $4.27 \pm 2.25$ & $4.02 \pm 3.22$ & & \\
\hline Gestation times & $3.25 \pm 2.07$ & $3.09 \pm 3.01$ & & \\
\hline \multicolumn{5}{|l|}{$\begin{array}{l}\text { Location of the } \\
\text { mixed tumor }\end{array}$} \\
\hline Upper & $53.79 \%(71 / 132)$ & $16.89 \%(37 / 219)$ & 52.625 & $<0.001$ \\
\hline Middle & $18.94 \%(25 / 132)$ & $33.79 \%(74 / 219)$ & & \\
\hline Lower & $27.27 \%(36 / 132)$ & $49.32 \%(108 / 219)$ & & \\
\hline $\begin{array}{l}\text { Uterine } \\
\text { leiomyoma }\end{array}$ & $4.55 \%(6 / 132)$ & $6.85(15 / 219)$ & 0.777 & 0.378 \\
\hline Atrophic vagina & $61.36 \%(81 / 132)$ & $57.08(125 / 219)$ & 0.624 & 0.430 \\
\hline HPV Positive & $0(0 / 132)$ & $70.78(155 / 219)$ & 167.306 & $<0.001$ \\
\hline $\begin{array}{l}\text { Operation } \\
\text { history of vulvar }\end{array}$ & $26.52(35 / 132)$ & $23.74 \%(52 / 219)$ & 0.339 & 0.560 \\
\hline $\begin{array}{l}\text { Malignancy } \\
\text { history }\end{array}$ & $2.27 \%(3 / 132)$ & $2.74(6 / 219)$ & 0.072 & 0.789 \\
\hline Hypertension & $12.12 \%(16 / 132)$ & $12.79 \%(28 / 219)$ & 0.033 & 0.856 \\
\hline $\begin{array}{l}\text { Diabetes } \\
\text { Mellitus }\end{array}$ & $15.15 \%(20 / 132)$ & $15.98 \%(35 / 219)$ & 0.043 & 0.836 \\
\hline Obesity & $38.64 \%(51 / 132)$ & $36.99 \%(81 / 219)$ & 0.096 & 0.757 \\
\hline
\end{tabular}

The average age of vulvar cancer with squamous intraepithelial lesion history while without vulvar nonneoplastic epithelial disorders history was $50.2 \pm$ 15 years old, which was slightly lower than vulvar cancer with vulvar nonneoplastic epithelial disorder history (51.9 \pm 16 years old). The average age of nonneoplastic epithelial disorders group is 45.9 years old, similar with the previous reports (44.5 years old). The average age of vulvar nonneolastic epithelial disorders group was obviously lower than vulvar cancer, which is different with previous reports that patients with vulvar nonneoplastic epithelial disorders were around $70 \pm 15$ years old. Although this difference was not statistically significant, it still indicated that two different kinds of mechanism exist in vulvar cancer development. Interesting, our study further showed the age distribution of vulvar cancer cases derived from vulvar nonneoplastic epithelial disorders. The data indicated that age distribution of vulvar cancer derived from vulva noneoplastic epithelial disorder has the similar trends with vulvar nonneoplastic epithelial disorders. On the contrary, different results showed the age distribution of vulvar cancer cases derived from HSIL. The data showed that age distribution of vulvar cancer derived from HSIL is similar with both LSIL and HSIL, indicating the strong connection between those diseases.

\section{The epidemiological features of vulvar nonneoplastic epithelial disorder, vulvar squamous intraepithelial lesion and vulvar cancer}

The lesion location of vulvar cancer was mainly distributed in the upper labium majora, labium minora and clitoris with the urethra as the boundary $(P<0.001)$ compared to the lesion location of most vulvar nonneoplastic epithelial disorder which was in the lower part. The significant difference of lesion sites between vulvar cancer and vulvar nonneoplastic epithelial disorders indicated that vulvar cancer and vulvar nonneoplastic epithelial disorder might have different pathogenesis. Even so, it was still an undisputable fact that quite a few patients who suffered vulvar nonneoplastic epithelial disorders before the onset of vulvar cancer. Further studies are 
needed to figure out the mechanism accounting for this difference in sites of lesions. Our analysis also showed that the proportion of the patients with low education level, atrophic vagina history and smoking are at higher risk of vulvar cancer, which was in accordance with previous reports. Interestingly, our analysis showed that patients who had sexual intercourse at a younger age were more susceptible to vulvar cancer $(p<0.001)$. The average age of those patients suffered vulvar cancer is much older than the average age of the patients with vulvar nonneoplastic epithelial disorder $(P<0.001)$. Their average delivery frequency are much more than those of the patients with vulvar nonneoplastic epithelial disorder. The types of pathological tissue of vulvar cancer and vulvar nonneoplastic epithelial disorder were analyzed. We found 102 patients with vulvar cancer have lichen simplex chronicus, accounting for $77.27 \%$ of the total vulvar cancer cases which resulted from noneoplatic epithelial disorder, which indicated the close relationship between above two diseases. This results was consistent with previous reports that lichen simplex chronicus was the main pathological type of vulvar cancer $(80.5 \%) .18$ cases with vulvar cancer have lichen scleroses, accounting for $13.64 \%$ of the total. There was a slight difference in the proportion of the patients with vulvar lichen scleroses and other vulvar disorders. Vulvar lichen scleroses accounts for a small proportion of vulvar cancer, about 5.7 times less than lichen simplex chronicus.

We compared above 4 groups using the adjustment formula $m=C_{k}^{2}=\frac{k(k-1)}{2}, \mathrm{k}$ represented group number, $\alpha^{\prime}=1-\sqrt[m]{1-\alpha}, \quad \alpha=0.0085$ (after adjusted).

HPV infection and genotypes in vulvar nonneoplastic epithelial disorders, vulvar squamous intraepithelial neoplasia and vulvar cancer

In this reports, we showed the distribution of high- and low-risk HPV types in 885 cases of vulvar diseases. Pathological and clinical variables were assessed by t-test, univariate odds ratios and logistic regression analysis. Generally, around $70 \%$ of vulvar cancers were HPV-positive. vulvar cancer with vulvar nonneoplastic epithelial disorder history had a higher average age but low HPV-positive ratio (0\%). Vulvar cancer with HSIL disorder history had a lower average age but extremely high ratio of HPV infection $(70.78 \%)$. The difference was statistically significant $(p<0.05)$. HPV16 was most common serotype in vulvar cancer or in vulvar squamous intraepithelial lesion. Other HPV types like HPV33 and HPV45 were less frequent. Low-risk HPV were uncommon in vulvar cancer. Tumors associated with any HPV type, any high-risk type or type 16 alone were less likely to metastasize than HPV-negative tumors. The incidence of HPV infection in vulvar cancer with vulvar nonneoplastic epithelial disorder was lower than vulvar cancer with HSIL disorder, which suggests a possibility that HPV infection is more closely linked with vulvar intraepithelial neoplasia than with nonneoplastic epithelial disorders.

\section{The incidence of vulvar nonneoplastic epithelial disorders, vulvar squamous intraepithelial lesion and vulvar cancer from 2006 to 2016}

The incidence of vulvar cancer in China in 2006 is 2.5/100,000, which is higher than the reports of Asia population $(0.3 / 100,000)$. The incidence is similar to the data of both Germen $(2-7 / 100,000)$ and America $(1.35-1.5 / 100,000)$. Around 27,000 women in China were diagnosed with vulvar cancer with a $50 \% 5$-year survival rate. The incidence of vulvar squamous intraepithelial lesion is round.7.0/100,000, which is higher than the incidence of vulvar cancer $[6,13]$. According to the population standard in Sichuan of China (in 2006), the incidence is 5.7/100,000. The incidence of vulvar nonneoplastic epithelial disorder is $1 / 300-1 / 1000$ [14]. By examining the patients' information during the past 10 years in our hospital, we found an increasing trend of the incidence of vulvar nonneoplastic epithelial disorders, vulvar squamous intraepithelial lesion and vulvar cancer. The incidence of vulvar squamous intraepithelial lesion doubled during the study period, which is similar to what reported by others.

\section{Diagnosis Time, recurrence rate and survival rate of vulvar cancers associated with different types of vulvar nonneoplastic epithelial disorders}

The previous analysis showed that the incidence of vulvar cancer was different in different pathological types of vulvar nonneoplastic epithelial disorders. Normally, cancerogenic probability of vulvar nonneoplastic epithelial disorders is about $3-5 \%$. The pathological type of lichen simplex chronicus is more likely resulted in vulvar cancer with high rate of $4-6 \%$. Vulvar squamous intraepithelial lesion is a major risk factor leading to vulvar cancer and regarded as precancerous lesion. According to the previous data, the cancerogenic probability is $20-30 \%$ [15]. In the later supplementary analysis, we visited 47 stage III vulvar cancer patients with nonneoplastic epithelial disorders. The missed follow-up rate is $14.90 \% .34$ vulvar cancer patients had lichen simplex chronicus and 6 patients had vulvar cancer resulted from vulvar lichen scleroses. The follow-up time 
ranges from 3 months to 15 years. With the diagnosis time as the beginning, we worked out the survival rate of patients. Table 5 shows the difference in the diagnosis time, recurrence rate and survival rate. Our analysis indicated that patients with vulvar lichen scleroses had worse prognosis. The survival rate of patients with high or low histological differentiation is $61.3 \%$ and $43.5 \%$ separately $(P<0.05)$, which is in accordance with other reports [16]. If the patients have vulvar nonneoplastic epithelial disorders before having vulvar cancer, their pathological type of vulvar nonneoplastic epithelial disorders will predict not only the probability of having vulvar cancer but also the prognosis of tumor.

Table 5. Diagnosis time, recurrence rate and survival rate of vulvar cancer

\begin{tabular}{|c|c|c|c|}
\hline $\begin{array}{l}\text { Type of } \\
\text { pathological tissue }\end{array}$ & $\begin{array}{l}\text { Lichen simplex } \\
\text { chronicus }\end{array}$ & $\begin{array}{l}\text { vulvar lichen } \\
\text { scleroses }\end{array}$ & $P$ Value \\
\hline $\begin{array}{l}\text { Diagnosis time } \\
\text { before } \\
\text { operation(median), } \\
\text { years }\end{array}$ & 10.6 & 6.2 & $P<0.05$ \\
\hline 3-year survival rate & $79.4 \%(27 / 34)$ & $50 \%(3 / 6)$ & $\begin{array}{l}P<0.05 \\
\text { Fisher exact } \\
\text { probability } \\
P=0.153\end{array}$ \\
\hline 5 year survival rate & $70.6 \%(24 / 34)$ & $50 \%(3 / 6)$ & $\begin{array}{l}P<0.05 \\
\text { Fisher exact } \\
\text { probability } p=0.370\end{array}$ \\
\hline Recurrence rate & $44.1 \%(15 / 34)$ & $33.3 \%(2 / 6)$ & $\begin{array}{l}P<0.05 \\
\text { Fisher exact } \\
\text { probability } p=1.000\end{array}$ \\
\hline
\end{tabular}

\section{Discussion}

Patients with vulvar squamous intraepithelial lesion have higher HPV infection rate than those with vulvar cancer. The relationship between HPV infection and gynecological tumor has always been the focus of studies [17]. It has been reported that one third of vulvar cancers have connection with HPV infection. HPV infection rate of women younger than 35 years old is about $85 \%$ [18]. E6 albumen, the highly hazardous HPV16/18 gene offspring can make P53 albumen dissolve and finally lead to the malignant change of cells [19]. Our study shows that HPV infection rates are different among vulvar carcinoma with vulvar nonneoplastic epithelial disorders history $(0 \%, 0 / 132)$, vulvar carcer with HSIL disorder history $(70.78 \%, 155 / 219)$, vulvar squamous intraepithelial lesion $(83.74 \%, 206 / 246)$, vulvar nonneoplastic epithelial disorders $(17.71 \%, 51 / 288)$ and Paget's tumor $(11.11 \%, 4 / 36)$. The difference in HPV infection rates, lesion sites and age further validates the hypothesis that vulvar squamous intraepithelial lesion and certain types of vulvar cancer have different epidemiological features. The vulvar cancers with negative HPV and positive HPV infection have different genetic mechanisms and different pathogenesis. It has been proposed during the recent years that P15 immunostaining can separate vulvar cancer and vulvar precancerous lesion which is connect with HPV infection [11].

Just as other malignancies, vulvar cancer is regulated by both genetic and epigenetic factors. It is also heavily influenced by life style especially those related to HPV infection. Exposure to carcinogens disrupts DNA replication and repair, and initiates the process of carcinogenesis. Particularly we find that age over 50 years old, early first coital age, atrophic vagina history, low educational level, smoking, with lichen simplex chronicus the pathological tissue type and with the upper vulvae as the location of pathology are predisposing factors of vulvar cancer. Symptoms such as nonpalpable lymphadenectasis of ilioinguinal lymph, pruritus vulvae resistant to treatments, location of pathology being in the clitoris or upper labium and vulvar mass with ulcer are warning signs of vulvar cancer. It is recommended that precautious measures be taken in patients with those symptoms. The hazardous factors for vulvar intraepithelial neoplasia were reported in a previous study, compared to which, our analysis showed that urethritis or colpitis history, easily agitated emotion, and multiple births are common hazardous factors for vulvar nonneoplastic epithelial disorder and vulvar cancer. Such factors as advanced age, nonpalpable lymphadenectasis of ilioinguinal lymph, upper vulvar atrophy pathology and with vulvar squamous epithelial hyperplasia as the pathological tissue type are the high risk factors leading to vulvar cancer [20].

The age distributions of the vulvar cancer group and other reference groups are discussed. Our analysis finds that the average age for vulvar nonneoplastic epithelial disorder is 45.9 years old and the youngest age is 23 years old, while the average age for vulvar cancer is 51.9 years old and the youngest age is 30 years old. The average age of vulvar cancer is higher than that of vulvar nonneoplastic epithelial disorder. The incidence of vulvar cancer is the highest between 50 to 59 years of age. It had been reported that over $80 \%$ of vulvar cancer patients were over the age of 50 years and over $50 \%$ were over the age of 70 years, which indicated that the incidence of vulvar caner peaks in females was 70 years and older [12]. It was unclear what account for the difference in peak incidence age between our observation and prior reports. It was noted that our study included only Chinese population, whereas the prior reports were based primarily on Caucasians. Being the more recent study, possible increases in HPV infection and/or 
other sexual transmitted diseases compared to early study populations could also account for the shift to younger patients. Other potential confounding factors for the difference may include sample size, increase awareness, early and more sensitive diagnosis of vulvar cancer. Further studies are needed to confirm our observation of earlier onset in Chinese population and explore explanations.

Sexual intercourse at young age and too many sexual partners were once considered as highly hazardous factors leading to vulvar cancer. Those are associated with HPV infection as well as hormone levels [21]. The recent analyses proved that HIV and HSV virus were also hazardous factors of vulvar cancer [22].

Smoking is a hazardous factor leading to various tumors especially lung cancer and alimentary tract tumor. Our analysis shows that, among patients with vulvar nonneoplastic epithelial disorder, smokers have a higher rate of vulvar cancer than the non-smokers possibly because there is a large amount of carcinogen in the tobaccos which can place a premium on P16 mutation in the cell. P16 is a multiple tumor suppressor. As an inhibitory factor of cyclins dependent kinase CDK4 and CDK6, it plays a negative-regulating role against the multiplication of cells by inhibit the positive function of Cyclin D1 on cell division. If the positive and negative regulating factors which regulate the normal growth of cell are disrupted, the cells will be proliferated abnormally and tumors will be induced. A study on vulvar cacotrophia and the expression of p16 and EGFR in the vulvar squama cancer found that p16 in GradeIII squama cancer was poorly expressed while RGFR excessively expressed. Thus it suggests that p16 is related to the malignant change of vulvar nonneoplastic epithelial disorder [23]. Our analysis also shows that patients with only vulvar nonneoplastic epithelial disorder have a smoking rate of $14.58 \%(42 / 288)$ and that of patients with vulvar cancer is $27.27 \%(36 / 132)$.

Family history of vulvar cancer is also a significant risk factor. It is reported that patients who have vulvar nonneoplastic epithelial disorder and family cancer history are more likely to have malignant change. In a family with multiple individuals affected by vulvar cancer, it is more likely that genetic mutations in cancer related genes are responsible. In addition similar living condition and dietetic habit can also contribute to tumorgenesis.

Early diagnosis and intervention is of great importance for patients with vulvar nonneoplastic epithelial disorders. It may take as long as 6.2 to10.6 years from diagnosis of vulvar nonneoplastic epithelial disorders to the onset of vulvar cancer. The time to give treatment is often delayed due to the uncertainty of whether vulvar nonneoplastic epithelial disorders would ultimately lead to vulvar cancer.

Our study shows an increasing trend of the incidence of vulvar nonneoplastic epithelial disorders and vulvar cancer. Approximately $5 \%$ of patients with vulvar nonneoplastic epithelial disorders may develop into vulvar cancer. A survey in 2006 showed that the incidence and death rate of vulvar cancer were both growing in the United States. In our study, we found a similar increase in the number of vulvar cancer and other vulvar diseases over the last decade. Furthermore, we found that in our study cohort the majority of vulvar cancer patients were 50-60 years old which is different from previous reports of most vulvar cancer patients are over the age of 70 years old. We have confirmed predictive value of risk factors such as HPV infection, age and first coital age in Chinese population. This study offers insights in prevention and treatment guideline development for vulvar cancer and future clinical trials.

\section{Acknowledgement}

The present study was supported by the Fund of the National Nature Science Foundations-National Excellent Young Scientists Fund grant (81001159); The Sichuan Province Science and Technology Plan Project (2014JY0213); The Scientific Research Foundation of Sichuan University for Outstanding Young Scholars (2013SCU04A22); The seventh new century education reform projects sponsored by Sichuan University (SCUY7093); The science benefiting demonstration project of Chengdu City (2015-HM02-00075-SF).

\section{Competing Interests}

The authors have declared that no competing interest exists.

\section{References}

1. Benedet JL, Bender H, Jones H, 3rd, Ngan HY, Pecorelli S. FIGO staging classifications and clinical practice guidelines in the management of gynecologic cancers. FIGO Committee on Gynecologic Oncology. International journal of gynaecology and obstetrics: the official organ of the International Federation of Gynaecology and Obstetrics. 2000; 70: 209-62.

2. Jones RW, Rowan DM, Stewart AW. Vulvar intraepithelial neoplasia: aspects of the natural history and outcome in 405 women. Obstetrics and gynecology. 2005; 106: 1319-26.

3. Mitchell MF, Prasad CJ, Silva EG, Rutledge FN, McArthur MC, Crum CP. Second genital primary squamous neoplasms in vulvar carcinoma: viral and histopathologic correlates. Obstetrics and gynecology. 1993; 81: 13-8.

4. Anastasiadis P, Skaphida P, Koutlaki N, Boli A, Galazios G, Liberis V. Trends in epidemiology of preinvasive and invasive vulvar neoplasias. 13 year retrospective analysis in Thrace, Greece. Archives of gynecology and obstetrics. 2000; 264: 74-9.

5. Deruelle P, Deruelle-Khazaal R, Collinet P, Lucot JP, Thomas P, Leroy JL. [Clinical study and prognosis of 56 cases of vulvar intraepithelial neoplasia]. Gynecologie, obstetrique \& fertilite. 2005; 33: 755-61.

6. Edwards CL, Tortolero-Luna G, Linares AC, Malpica A, Baker VV, Cook E, et al. Vulvar intraepithelial neoplasia and vulvar cancer. Obstetrics and gynecology clinics of North America. 1996; 23: 295-324. 
7. Proceedings of the Eighth World Congress of the International Society for the Study of Vulvar Disease. Mariehamn, Finland, September 9-15, 1985. The Journal of reproductive medicine. 1986; 31: 773-838.

8. Iwasawa A, Nieminen P, Lehtinen M, Paavonen J. Human papillomavirus in squamous cell carcinoma of the vulva by polymerase chain reaction. Obstetrics and gynecology. 1997; 89: 81-4.

9. Ansink AC, Heintz AP. Epidemiology and etiology of squamous cell carcinoma of the vulva. European Journal of Obstetrics Gynecology \& Reproductive Biology. 1993; 48: 111-5.

10. Kaufman RH. Intraepithelial neoplasia of the vulva. Gynecologic oncology. 1995; 56: 8-21.

11. Trimble CL, Hildesheim A, Brinton LA, Shah KV, Kurman RJ. Heterogeneous etiology of squamous carcinoma of the vulva. Obstetrics and gynecology. 1996; 87: 59-64.

12. Wagner W, Prott FJ, Weissmann J, Niewohner-Desbordes U, Ostkamp K, Alfrink M. Vulvar carcinoma: a retrospective analysis of 80 patients. Archives of gynecology and obstetrics. 1999; 262: 99-104.

13. Vilmer C, Cavelier-Balloy B, Nogues C, Trassard M, Le Doussal V. Analysis of alterations adjacent to invasive vulvar carcinoma and their relationship with the associated carcinoma: a study of 67 cases. European journal of gynaecological oncology. 1998; 19: 25-31.

14. Wang J CY, Hang LX. The clinic analysis and prognosis of vulvar carcinoma. Journal of Chinese physician. 2004; 6: 680-1.

15. McNally OM, Mulvany NJ, Pagano R, Quinn MA, Rome RM. VIN 3: a clinicopathologic review. International journal of gynecological cancer : official journal of the International Gynecological Cancer Society. 2002; 12: 490-5.

16. Rosen C, Malmstrom H. Invasive cancer of the vulva. Gynecologic oncology. 1997; 65: 213-7.

17. Weaver BA. Epidemiology and natural history of genital human papillomavirus infection. The Journal of the American Osteopathic Association. 2006; 106: S2-8.

18. Al-Ghamdi A, Freedman D, Miller D, Poh C, Rosin M, Zhang L, et al. Vulvar squamous cell carcinoma in young women: a clinicopathologic study of 21 cases. Gynecologic oncology. 2002; 84: 94-101.

19. Scheffner M, Munger K, Byrne JC, Howley PM. The state of the p53 and retinoblastoma genes in human cervical carcinoma cell lines. Proceedings of the National Academy of Sciences of the United States of America. 1991; 88: 5523-7.

20. Tang GX, Wu X, Chen JP, Zhou BS. [Study on the risk factors of 100 cases with vulvar dystrophy]. Zhonghua liu xing bing xue za zhi $=$ Zhonghua liuxingbingxue zazhi. 2003; 24: 932-4.

21. Baseman JG, Koutsky LA. The epidemiology of human papillomavirus infections. Journal of clinical virology : the official publication of the Pan American Society for Clinical Virology. 2005; 32 Suppl 1: S16-24.

22. Jamieson DJ, Paramsothy P, Cu-Uvin S, Duerr A. Vulvar, vaginal, and perianal intraepithelial neoplasia in women with or at risk for human immunodeficiency virus. Obstetrics and gynecology. 2006; 107: 1023-8.

23. Zhang JG YC, Liu CH, et al. Significance and expression of p16 and EGFR in vulvar dystrophy and squamous cell carcinoma of vulva. Journal of Fourth Mil Med University. $200425: 445$. 